\title{
Infant mental health: classification and relevance for clinicians
}

\author{
Boolang R. Ahamat, ${ }^{1}$ Helen Minnis ${ }^{2}$
}

The Psychiatrist (2012), 36, 230-234, doi: 10.1192/pb.bp.110.033969

${ }^{1} \mathrm{NHS}$ Greater Glasgow and Clyde, Glasgow, UK; ${ }^{2}$ University of Glasgow, UK

Correspondence to Helen Minnis (helen.minnis@glasgow.ac.uk)

First received 21 Jan 2011, final revision 3 Nov 2011, accepted 12 Jan 2012
Summary Infant mental health is a growing research area, but findings have not generally translated into new service developments. Recognising mental health problems in young infants is relevant for clinicians in all mental health specialties, but it can be a particular challenge to make diagnoses in very young children. Mental health classification systems are fraught with the difficulties of standardising diagnoses for infants, while trying to provide a clinically useful and relevant framework. The diagnostic classification DC:0-3 appears to have strengths, for example, a clear space to consider relationship disorders, and therefore encouraging a broad assessment of the child and family. More information is beginning to gather regarding infant mental health services around the world and assessment of this patient group in clinical practice. This commentary aims to help inform clinicians about this developing field.

Declaration of interest None.
Some understanding of infant mental health is essential for all psychiatrists: in adult psychiatry, forensic psychiatry, addictions and learning disability psychiatry, patients' difficulties can have a profound impact on their relationship with their young children and a significant impact on the child's future development. Problems presenting in early childhood can signal infant mental illness, infant-carer relationship difficulties, or be an early warning for recognised syndromes in middle childhood. ${ }^{1}$ This is becoming more relevant to psychiatric services worldwide, as intervention in the early years may be financially sensible $^{2}$ (Fig. 1) and alter the prognosis of affected children. ${ }^{3}$ Figure 1 represents educational interventions for children, but its message is equally relevant for health: it has been amply demonstrated that focused, evidence-based interventions delivered in the early years can have a profound and long-lasting effect on a range of outcomes including crime, substance use, sexual behaviour and earnings. ${ }^{4,5}$

Problems in the early months and years of life are related to a range of difficulties across the lifespan, including conduct disorder/antisocial personality disorder, suicide and cardiovascular disease ${ }^{6,7}$ For example, children with early-onset persistent aggression have escalating problems - in adolescence, they already have nine times the risk of death from all causes. ${ }^{8}$ Societal costs will be ten times those of their peers who do not exhibit aggressive behaviour. ${ }^{9}$ Effective early interventions and preventive programmes which target a range of difficulties now exist. ${ }^{10-13}$ However, despite increased understanding of the importance of infant mental health, this has not translated into service development. This may, at least in part, be because clinicians lack the confidence and the tools to deliver infant mental health assessments and treatments.

Despite an explosion in research on infant mental health in recent years, it has not been easy to translate research findings into clinical practice: a review by Skovgaard et $a l^{14}$ highlights the gaps in the literature regarding infant mental health, in particular that research generally uses small samples of high-risk children and families, and relationship assessments used may be expensive and time consuming. We were curious to see what the literature has identified as key issues in infant mental health - of use for clinicians - and what measures were being used clinically to classify disorders in this age range.

\section{What might infant mental health services look like?}

There is a paucity of research on infant mental health based in clinical settings. One clinical study noticed that the majority of referrals from a high-risk, socioeconomically deprived population fell within the third year of life, and speculated that clinicians tend not to refer before the age of 1 year. ${ }^{15}$ They noticed a higher rate of classified infant mental health disorders such as post-traumatic stress disorder (PTSD), regulatory disorder and multisystem developmental disorder ${ }^{16}$ from 25 to 36 months compared with earlier - perhaps related to the fact that symptoms of these disorders may only be unmasked when the infant moves through the developmental changes during this time. ${ }^{15}$ The Diagnostic Classification of Mental Health and Developmental Disorders of Infancy and Early Childhood 


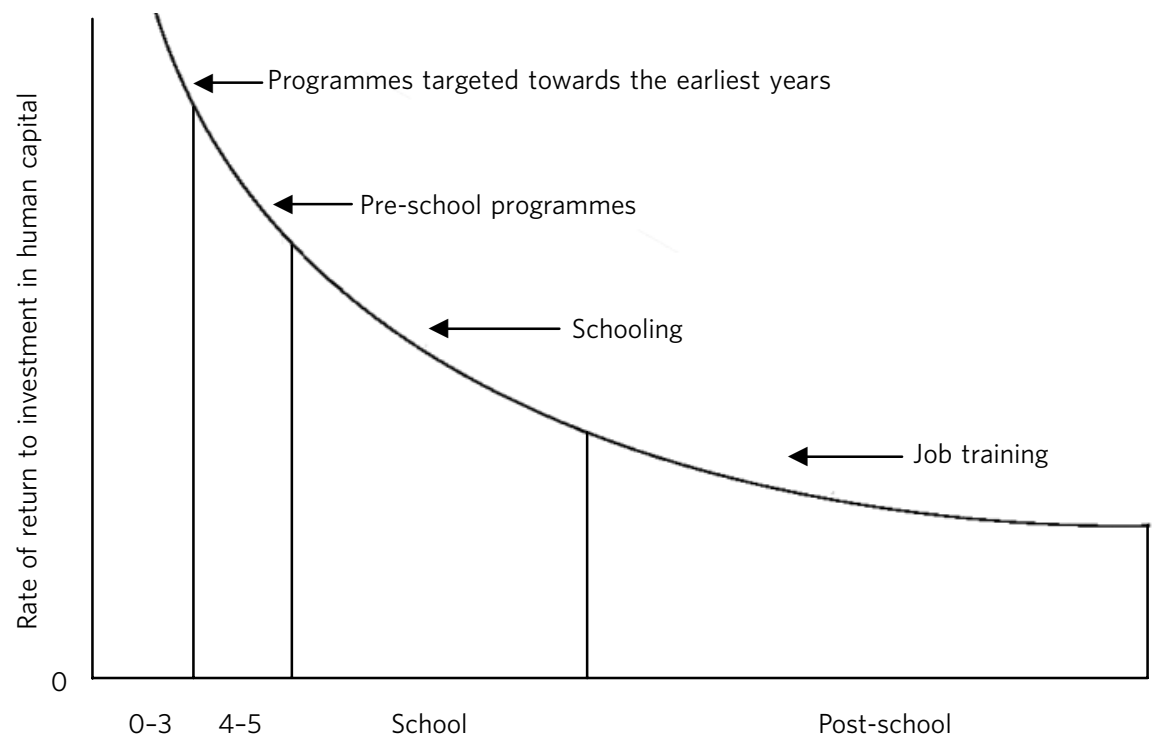

Fig. 1 Returns to a unit dollar invested. Reproduced with permission from Heckmann. ${ }^{2}$

(DC:0-3) axis I diagnoses are more frequently made from the age of 3 years, with an increase in diagnoses of neurodevelopmental disorders and a decrease in feeding and sleeping disorders compared with the first year of life: ${ }^{17}$ this seems sensible given the developmental changes which occur in this time. However, the increase in referrals from the second year of life could hint at a critical developmental/vulnerable period at this time where developmental capacities may deviate from the norm and parents seek advice. ${ }^{17}$

The most common symptoms found in referred infants, in clinical studies, were sleep problems ${ }^{18}$ and eating problems. ${ }^{19}$ The vast majority of referrals were due to a concern noted about the child, with only a minority of families referred due to a primary relationship problem identified by the referrer. ${ }^{19,20}$

Regarding interventions, a meta-analysis by BakermansKranenburg et $a l^{10}$ described that 'less is more' when it comes to interventions for improving the parent-infant relationship and attachment security: they found that the most successful interventions did not depend on large numbers of sessions. Their findings demonstrated that the idea of 'any intervention being good intervention' is also not necessarily the case: an intervention with a modest number of sessions $(<16)$ and clear behaviour focus for parents seems most useful.

\section{Classifying infant mental health problems}

'Epidemiology has demonstrated that early childhood disorders are as impairing, persistent, and associated with known psychopathology risk factors as disorders at other points in childhood'. ${ }^{21}$ Despite the importance of identifying mental health problems in very young children, applying DSM criteria is challenging because of the rapid developmental changes, limited language abilities and interdependence with caregivers. $^{21,22}$ Some DSM-IV diagnoses can be applied to, and are relevant for, very young children, and clinicians are encouraged to use DSM-IV diagnoses where appropriate. ${ }^{21}$ Gillberg ${ }^{1}$ has coined an acronym, ESSENCE (Early Symptomatic Syndromes Eliciting Neurodevelopmental Clinical Examinations), which addresses some of the challenges faced by clinicians when categorically diagnosing disorders in pre-school children. He suggests that certain symptoms can act as markers for a neurodevelopmental disorder later in childhood. He highlights the potential pitfalls of making a definitive diagnosis in the pre-school age group where such overlap among disorders exists, and counsels that separation of disorders may be premature and clinically unhelpful.

In contrast, others have found it useful to classify disorder in very young children; Egger \& Emde $^{21}$ have provided a detailed review of the development of criteria for classifying mental health disorders in early childhood. The DC:0-3 tool was developed in 1994 to complement existing classification systems and to provide a clear clinical framework for diagnosis up to age 4. In contrast, the Research Diagnostic Criteria - Preschool Age (RDC-PA) offered another diagnostic classification, heavily weighted towards DSM-IV and promoting systematic research into infant mental health by offering clearly specified criteria. $^{23}$ The DC:0-3 tool was subsequently revised in 2005 (DC:0-3R) ${ }^{16}$ in an attempt to combine learning from the more 'top-down' RDC-PA approach that begins with classifications used in older children and adults and the DC:0-3 approach that uses scientific knowledge specific to infants and young children. ${ }^{21}$ Although similar to the RDC-PA via common DSM-IV traits, DC:0-3R is more clinically oriented and has been used widely in clinical practice with children up to the age of 5 years. ${ }^{18}$

The DC:0-3R tool is a multi-axial categorical classification system with five axes: axis I, primary diagnosis; axis II, relationship disorder classification; axis III, medical and developmental disorders and conditions; axis IV, 
psychosocial stressors; and axis V, emotional and social functioning. ${ }^{16}$ Axes I, III and IV are similar to those in the DSM-IV. Axis II is a new way of including the parent-infant relationship, which may be disordered. Emde \& Wise ${ }^{24}$ also suggested a sixth axis to include consideration of family history of mental disorder, availability of family resources and cultural factors.

The DC:0-3 manual includes advice on the use of its system, and explains various assessment tools used to help the clinician classify a disorder. Wright $\&$ Northcutt $^{25}$ also developed some 'decision trees' to help guide the clinician with questions to ask in clinical assessments, observation of the parent-infant interactions, and assessment of the functional emotional developmental level, with reference to DC:0-3.

When looking at the relationship between DC:0-3 and DSM classifications, similarities exist between axis I disorders such as PTSD, adjustment disorder, ${ }^{26}$ affective disorders, disorders of sleep and eating ${ }^{24}$ and reactive attachment disorder (RAD) ${ }^{18,26}$ (reactive attachment disorder has been revised to deprivation/maltreatment disorder in DC:0-3R, due to confusion between attachment problems which could be included in axis $\mathrm{II}^{16}$ ).

\section{What are regulatory disorders?}

Regulatory disorders have not been previously described in any other classification system. Several subtypes exist in DC:0-3. These disorders refer to the difficulties a child may have in regulating emotions and behaviours in response to sensory stimulation, leading to impaired development and functioning, ${ }^{16}$ thought to share some common ground with the concept of temperament, where disorder occurs at the extremes. ${ }^{14}$ The subtypes include hypersensitive, fearful/ cautious, negative/defiant, hyposensitive/underresponsive and sensory stimulation-seeking/impulsive. ${ }^{16}$ Early use of DC:0-3 showed that paediatricians found these terms useful to describe very young $(<1$ year old) children who do not suit DSM criteria. ${ }^{26}$ There has been overlap identified with DC:0-3 regulatory disorder and DSM diagnoses of oppositional defiant disorder (ODD) and attention-deficit hyperactivity disorder (ADHD); for example, in one study $13 / 20$ infants diagnosed with regulatory disorder could also receive a DSM diagnosis of ODD or ADHD. ${ }^{18}$

\section{The importance of axis II}

The inclusion of an entire axis in DC:0-3 to describe relationship disorders, with a proposed assessment tool, the Parent Infant Relationship - Global Assessment Scale (PIRGAS), allows assessment of relationship problems which may not have been addressed in any other evaluation of the child. ${ }^{15,24,27}$ It widens the thinking beyond the child, concentrating somewhere between the 'child with the problem' and the caregiver potentially as the 'cause' of the problem, and guides clinicians to investigate this complex relationship in a standardised and purposeful way. ${ }^{15}$ There already exist many ways to consider the infant-caregiver relationship, and it can be hard to distinguish the phenomenology in terms of relationship qualities, attachment and other descriptive concepts such as temperament. With regard to the attachment relationship, maternal sensitivity has been traditionally seen as a focus of importance: ${ }^{28}$ maternal insensitivity can be related to mental health outcomes in children, particularly aggression. ${ }^{29}$ Temperament in infancy has also been related to childhood mental illness. ${ }^{30}$

Attachment disorders are placed in axis I because they are pervasive disorders identified within the child. In contrast, relationship disorders may be specific to the individual relationship between the parent-infant dyad. Maldonado-Duran et $a l^{15}$ consider how attachment disorders relate to relationship disorders: although attachment disorders are diagnosed in axis I where there has been historical evidence of maltreatment or deprivation, it may be argued that relationship disorders in infancy are closely related to the subsequent development of attachment disorders. There is also a question as to whether attachment disorders exist discretely, or whether attachment should be considered part of other disorders as an associated relationship disturbance. ${ }^{27}$ The DC:0-3R tool further clarifies that observation and evaluation of the current relationship is rated under axis II. The issue of the effect of inexperienced parents on the relationship has also been raised, although not included in DC:0-3. ${ }^{15}$

\section{Assessment tools for the $\mathbf{0 - 3}$ age range}

Assessments based on DSM-IV and DC:0-3 can still seem imperfect: there is a general lack of research into infant mental health disorders using DSM criteria, and a lack of reliability and validity using DC:0-3. ${ }^{30}$ Tools to assess infant mental health rely on observation of the infant and infant-carer relationship (PIR-GAS) and reports from informants, namely parents/carers (using the Child Behaviour Checklist (CBCL) ${ }^{31}$ and the Preschool Age Psychiatric Assessment (PAPA) ${ }^{32}$ ): infants communicate largely non-verbally and are therefore unable to report on their own symptoms. There have been attempts to standardise parent report questionnaires in this age range using the CBCL and the Strengths and Difficulties Questionnaire (SDQ; www.sdqinfo.com). The CBCL now has a version for children as young as 18 months, ${ }^{31}$ and the SDQ has been adapted for age 2-3 years (R. Goodman, personal communication, 2011).

Observation of the relationship between the parent/ primary caregiver and infant is essential for classification with DC:0-3. The description and guidance on the use of the PIR-GAS is detailed in DC:0-3R, along with another tool, the Relationship Problems Checklist (RPCL), to classify the quality of the relationship on axis II. $^{16}$ In practice, the interaction between infant and caregiver is commonly recorded on video, for subsequent scoring with the PIR-GAS. The PIR-GAS is scored out of 100, where 81-100 represents an adapted 'good enough' relationship; 41-80 suggests a relationship at risk of dysfunction, and 40 or less represents a disordered relationship (i.e. a relationship disorder which can be coded under axis II). ${ }^{16,33}$ There is consensus that the overall assessment of an infant and parent should be approached 
from an 'ecological' perspective, observing the child and parent in different settings to take into account any situational variations which may affect the child at the time of assessment. ${ }^{15}$ The PIR-GAS seems to be particularly valuable in detecting internalising symptoms. ${ }^{33,34}$

The parent-reported CBCL $1.5-5^{31}$ includes a language development screening tool to link whether a child with language problems may also have behavioural/emotional problems, and aims to maintain DSM-based diagnostic scales with seemingly good reliability and validity; its use is suggested as a preclinical assessment tool. The PAPA is a structured parent interview using DSM and DC:0-3 principles to diagnose children aged $2-5$ years, ${ }^{32}$ developed to further address the diagnostic challenges in young children. The Diagnostic Infant and Preschool Assessment (DIPA) ${ }^{35}$ is another caregiver-reported tool that follows DSM criteria to assess children aged $1-6$ years; it has shown good agreement in concurrent criterion validity to the CBCL 1.5-5 and is more concise than the PAPA.

\section{Reliability of diagnosis in infancy}

Several authors have compared and contrasted DC:0-3 with DSM or ICD systems. Frankel et $a l^{18}$ used both DSM-IV and DC:0-3 to diagnose the same sample of patients in their general infant patient group, and suggested good concordance between the two systems for axis I disorders. Another study looked at the reliability of use of ICD-10 and DC:0-3 in 1.5-year-old infants ${ }^{36}$ and found greater interrater reliability and test-retest reliability using DC:0-3, but demonstrated that ICD-10 in infants also provides good reliability. With regard to the PIR-GAS, there was $100 \%$ agreement between raters, suggesting it is a reliable assessment tool for axis II. ${ }^{36}$

Stafford $e t a l^{27}$ reviewed two diagnostically challenging conditions in young children (RAD and PTSD) and their classification in different systems. The authors comment that infants may present with PTSD symptoms but not fulfil DSM-IV criteria due to their non-verbal communication (i.e. not being able to describe the subjective experience), therefore DC:0-3 included more 'behaviourally anchored' criteria. The authors also favour DSM-IV for diagnosing RAD (as 'a within-child disorder'), due to the lack of criteria in DC:0-3, although they acknowledge the benefits of considering relationship problems in general as part of axis II of DC:0-3. When considering the symptoms of these disorders and how they present within certain developmental stages, Stafford et $a l^{27}$ criticise DC:0-3 for not accounting for age and developmental capabilities. As a result of these diagnostic challenges, some of the main changes from DC:0-3 to DC:0-3R include: changing RAD to deprivation/maltreatment disorder, separating it from possible axis II classifications regarding the attachment relationship; and consolidating PTSD as a disorder in DC:0$3 \mathrm{R}$, where traumatic stress disorder stood before, allowing more analogy with DSM-IV and adding in extra diagnostic criteria for infants. ${ }^{16}$ Another significant revision was the restricted use of multisystem developmental disorder to infants under the age of 2 years, accounting for the increase in research on autism spectrum disorders demonstrating a diagnosis can be made from the age of $2 .^{16,37}$ This could support the view that in infants younger than 2 years a broader view of neurodevelopmental disorders should be taken, in line with the ESSENCE concept. ${ }^{1}$

It may be that DC:0-3R and other infant assessment tools should marry with, and not replace, existing classification systems: the DC:0-3R, although filling in gaps with specific infant disorders not found in DSM and ICD systems, has a clinical focus and could be balanced by stricter research- and evidence-based approaches like the RDC-PA. ${ }^{38}$

\section{Implications for mental health services}

At the moment, generic child psychiatric services may be struggling to assess infants, despite a general interest in early intervention on the research and political levels, ${ }^{9}$ perhaps because services are overstretched to manage their existing child and adolescent patients. Early difficulties such as sleeping, behaviour or feeding problems often fall to health visitors to manage and child psychiatrists might feel inadequately trained in assessing and treating infant mental health problems. Interestingly, there is some evidence to suggest that infant mental health problems could be predicted during a routine general health surveillance carried out by non-mental health professionals in early infancy, potentially identifying infants at risk of mental health problems in early life. ${ }^{39}$ Perinatal services remain focused on maternal mental health as their main priority: ${ }^{40}$ although some monitoring of the mother-baby relationship occurs in an in-patient setting with mothers with the most severe illness, any out-patient work around this is generally performed by health visitors and social work agencies. Parenting capacity and child protection are paramount across psychiatry: Milburn et $a l^{41}$ highlight the process of assessment of these factors with parents and children of all ages, especially infants in vulnerable families, with consideration towards involvement of statutory measures where necessary. Infant mental health is just starting to reach the curriculum for child psychiatrists in training, although hands-on experience is lacking.

\section{About the authors}

Boolang R. Ahamat, Specialty Trainee (ST5) in Child and Adolescent Psychiatry, NHS Greater Glasgow and Clyde, Scotland, UK, and Helen Minnis, Senior Lecturer in Child and Adolescent Psychiatry, Mental Health and Well Being, College of Medicine, Veterinary Science and Life Sciences, University of Glasgow, Scotland, UK.

\section{References}

1 Gillberg C. The ESSENCE in child psychiatry: early symptomatic syndromes eliciting neurodevelopmental clinical examinations. Res Dev Disabil 2010; 31: 1543-51.

2 Heckman JJ. Schools, skills and synapses. Econ Inq 2008; 46: 289.

3 Wallace KS, Rogers SJ. Intervening in infancy: implications for autism spectrum disorders. J Child Psychol Psychiatry 2010; 51: 1300-20.

4 Olds D, Henderson CR Jr, Cole R, Eckenrode J, Kitzman H, Luckey D, et al. Long-term effects of nurse home visitation on children's criminal and antisocial behaviour: 15-year follow-up of a randomized controlled trial. JAMA 1998; 14: 1238-44. 
5 Schweinhart LJ, Montie J, Xiang Z, Barnett WS, Belfield CR, Nores M. Lifetime Effects: The High/Scope Perry Preschool Study through age 40. High/Scope Press, 2005.

6 Deans KA, Bezlyak V, Ford I, Batty GD, Burns H, Cavanagh J, et al. Differences in atherosclerosis according to area level socioeconomic deprivation: cross sectional, population based study. BMJ 2009; 339: $1-12$

7 Dube SR, Anda RF, Felitti VJ, Chapman D, Williamson DF, Giles WH. Childhood abuse, household dysfunction and the risk of attempted suicide throughout the life span: finding from the Adverse Childhood Experiences Study. JAMA 2001; 286: 3089-96.

8 Coffey C, Veit F, Wolfe R, Cini E, Patton GC. Mortality in young offenders: retrospective cohort study. BMJ 2003; 326: 1064.

9 Scott S, Knapp M, Henderson J, Maughan B. Financial cost of social exclusion: follow up study of antisocial children into adulthood. BMJ 2001; 323: 1-5.

10 Bakermans-Kranenburg MJ, van IJzendoorn MH, Juffer F. Less is more: meta-analyses of sensitivity and attachment interventions in early childhood. Psychol Bull 2003; 129: 195-215.

11 Scott S, Spender Q, Doolan M, Jacobs B, Aspland H, Webster-Stratton C. Multicentre controlled trial of parenting groups for childhood antisocial behaviour in clinical practice. BMJ 2001; 323: 194

12 Coolican J, Smith IM, Bryson SE. Brief parent training in pivotal response treatment for preschoolers with autism. J Child Psychol Psychiatry 2010; 51: 1321-30.

13 Havighurst SS, Wilson KR, Harley AE, Prior MR, Kehoe C. Tuning in to kids: improving emotion socialization practices in parents of preschool children - findings from a community trial. J Child Psychol Psychiatry 2010; 51: 1342-50.

14 Skovgaard AM, Houmann $T$, Landorph SL, Christiansen $E$ Assessment and classification of psychopathology in epidemiological research of children 0-3 years of age: a review of literature. Eur Child Adolesc Psychiatry 2004; 13: 337-46.

15 Maldonado-Duran M, Helmig L, Moody C, Fonagy $\mathrm{P}$, Lartigue $T$ Sauceda-Garcia JM, et al. The Zero-to-Three diagnostic classification in an infant mental health clinic: its usefulness and challenges. Infant Ment Health J 2003; 24: 378-97.

16 Zero to Three National Centre for Infants. Parent-Infant Relationship Global Assessment Scale (PIR-GAS) in DC:0-3R Diagnostic Classification of Mental Health and Developmental Disorders of Infancy and Early Childhood. Zero to Three, 2005.

17 Cordeiro MJ, Da Silva PC. Diagnostic classification: results from a clinical experience of three years with DC:0-3. Infant Ment Health $J$ 2003; 24: 349-64

18 Frankel KA, Boyum LA, Harmon RJ. Diagnoses and presenting symptoms in an infant psychiatry clinic: comparison of two diagnostic systems. J Am Acad of Child Adolesc Psychiatry 2004; 43: 578-87.

19 Keren M, Feldman R, Tyano S. Diagnoses and interactive patterns of infants referred to a community-based infant mental health clinic. J Am Acad of Child Adolesc Psychiatry 2001; 40: 27-35.

20 Knapp PK, Ammen S, Arstein-Kerslake C, Poulsen MK, Mastergeorge A Feasibility of expanding services for very young children in the public mental health setting. J Am Acad of Child Adolesc Psychiatry 2007; 46: 152-61.

21 Egger HL, Emde RN. Developmentally sensitive diagnostic criteria for mental health disorders in early childhood: the Diagnostic and Statistical Manual of Mental Disorders IV, the Research Diagnostic Criteria-Preschool Age, and the Diagnostic Classification of Mental Health and Developmental Disorders of Infancy and Early ChildhoodRevised. Am Psychol 2011; 66: 95-106.

22 American Psychiatric Association. Diagnostic and Statistical Manual of Mental Disorders (4th edn) (DSM-IV). APA, 1994.
23 Task Force on Research Diagnostic Criteria: Infancy and Preschool. Research diagnostic criteria for infants and preschool children: the process and empirical support. J Am Acad of Child Adolesc Psychiatry 2003; 42: 1504-12.

24 Emde RN, Wise BK. The cup is half full: initial clinical trials of DC:0-3 and a recommendation for revision. Infant Ment Health J 2003; 24: 437-46.

25 Wright C, Northcutt C. Schematic decision trees for DC:0-3. Infant Ment Health J 2004; 25: 171-4.

26 Dunitz M, Scheer PJ, Kvas E, Macari S. Psychiatric diagnoses in infancy: a comparison. Infant Ment Health J 1996; 17: 12-23.

27 Stafford B, Zeanah CH, Scheeringa M. Exploring psychopathology in early childhood: PTSD and attachment disorders in DC:0-3 and DSMIV. Infant Ment Health J 2003; 24: 398-409.

28 De Wolff MS, van ljzendoorn MH. Sensitivity and attachment: a metaanalysis on parental antecedents of infant attachment. Child Dev 1997; 68: 571-91.

29 Alink LRA, Mesman J, van Zeijl J, Stolk MN, Juffer F, BakermansKranenburg MJ, et al. Maternal sensitivity moderates the relation between negative discipline and aggression in early childhood. Soc Dev 2009; 18: 99-120.

30 Kagan J, Snidman N, Zentner M, Peterson E. Infant temperament and anxious symptoms in school age children. Dev Psychopathol 1999; 11: 209-24.

31 Rescorla LA. Assessment of young children using the Achenbach System of Empirically Based Assessment (ASEBA). Ment Retard Dev Disabil Res R 2005; 11: 226-37.

32 Egger HL, Angold A. The Preschool Age Psychiatric Assessment (PAPA): a structured parent interview for diagnosing psychiatric disorders in preschool children. In A Handbook of Infant, Toddler, and Preschool Mental Health Assessment (eds R Delcarmen-Wiggens, A Carter): 223-43. Oxford University Press, 2004.

33 Thomas JM, Guskin KA. Disruptive behaviour in young children: what does it mean? J Am Acad Child Adolesc Psychiatry 2001; 40: 44-51.

34 Aoki Y, Zeanah CH, Scott Heller S, Bakshi S. Parent-Infant Relationship Global Assessment Scale: a study of its predictive validity. Psychiatry Clin Neurosci 2002; 56: 493-7.

35 Scheeringa MS, Haslett N. The reliability and criterion validity of the diagnostic infant and preschool assessment: a new diagnostic instrument for young children. Child Psychiatry Hum Dev 2010; 41: 299-312.

36 Skovgaard AM, Christiansen E, Andreasen AH. The reliability of the ICD-10 and the DC:0-3 in an epidemiological sample of children $1 \frac{1}{2}$ years of age. Infant Ment Health J 2005; 26: 470-80.

37 Carr T, Lord C. Autism spectrum disorders. In Handbook of Infant Mental Health (ed. CH Zeanah Jr): 301-17. Guilford Press, 2009.

38 Postert C, Averbeck-Holocher M, Beyer T, Muller J, Furniss T. Five systems of psychiatric classification for preschool children: do differences in validity, usefulness and reliability make for competitive or complimentary constellations? Child Psychiatry Hum Dev 2009; 40: 25-41.

39 Skovgaard AM, Houmann T, Christiansen E, Olsen EM, Landorph SL, Lichtenberg $A$, et al. Can a general health surveillance between birth and 10 months identify children with mental disorder at $1 \frac{1}{2}$ year? A case-control study nested in cohort CCC 2000. Eur Child Adolesc Psychiatry 2008; 17: 290-8.

40 Austin M-P, Priest SR. Clinical issues in perinatal mental health: new developments in the detection and treatment of perinatal mood and anxiety disorders. Acta Psychiatr Scand 2005; 112: 97-104.

41 Milburn NL, Lynch M, Jackson J. Early identification of mental health needs for children in care: a therapeutic assessment programme for statutory clients of child protection. Clin Child Psychol Psychiatry 2008; 13: $31-47$ 